\title{
Primary aldosteronism and pregnancy
}

Authors: $\quad$ Zelinka Tomáš, Widimský Jiří, jr.

Hospital: 3rd Department of Medicine - Department of Endocrinology and Metabolism, First Faculty of Medicine, Charles University in Prague and General University Hospital in Prague

Primary aldosteronism (PA) may present in younger age and it may so complicate pregnancy if not diagnosed early. Our aim was to identify patients in whom PA was diagnosed after pregnancy and to seek for possible complications during pregnancy.

\section{Results:}

Retrospective analysis of female patients with PA treated at our institution in whom PA was diagnosed after pregnancy.

Eleven subjects with PA were identified. All subjects presented with profound hypokalemia a significantly increased aldosterone levels. In 10 cases, final diagnosis of aldosterone-producing adenoma was made, one subject presented with idiopathic aldosteronism.

Table 1 Baseline characteristics of subjects with primary aldosteronism

\begin{tabular}{|c|c|c|c|c|c|c|c|c|c|c|c|}
\hline Patient \# & $\begin{array}{l}\text { Age at } \\
\text { diagnosis } \\
\text { (years) }\end{array}$ & $\begin{array}{c}\text { Duration of } \\
\text { hypertension } \\
\text { (years) }\end{array}$ & $\begin{array}{l}\text { Severity of } \\
\text { hypertension }\end{array}$ & Symptoms & $\begin{array}{l}\text { Lowest } \mathrm{K}^{+} \\
(\mathrm{mmol} / \mathrm{l})\end{array}$ & $\begin{array}{l}\text { Aldosterone } \\
\qquad(\mathrm{ng} / \mathrm{dl})\end{array}$ & $\begin{array}{l}\text { Plasma renin activity/ } \\
\text { Plasma renin } \\
(\mu \mathrm{g} / / \mathrm{h} / / \mathrm{pg} / \mathrm{ml})\end{array}$ & $\begin{array}{c}\text { Final } \\
\text { diagnosis }\end{array}$ & $\begin{array}{l}\mathrm{K}^{+} \text {after } \\
\text { operation } \\
(\mathrm{mmol} / \mathrm{l})\end{array}$ & $\begin{array}{l}\text { Aldosterone after } \\
\text { operation (ng/dl) }\end{array}$ & $\begin{array}{c}\text { Hypertension after } \\
\text { operation }\end{array}$ \\
\hline 1 & 29 & 0.5 & severe & headache & 2.9 & 47.1 & 0.6 (PRA) & APA & 4.6 & 2.3 & mild \\
\hline 2 & 36 & 0.75 & moderate & 0 & 2.6 & 48.9 & $0.58(\mathrm{R})$ & APA & 3.8 & 4.7 & normotension \\
\hline 3 & 32 & 4 & mild & tiredness & 2.8 & 53.4 & 0.34 (PRA) & APA & 4.7 & 2 & normotension \\
\hline 4 & 39 & 6 & mild & tiredness, cramps & 2.4 & 38.6 & $3.3(\mathrm{R})$ & APA & 4.7 & 9.7 & normotension \\
\hline 5 & 38 & 12 & moderate & tiredness, cramps & 1.75 & 63.8 & $4.8(\mathrm{R})$ & APA & 5.1 & 3.4 & mild \\
\hline 6 & 33 & 6 & moderate & $\begin{array}{l}\text { tiredness, ankle } \\
\text { swellings }\end{array}$ & 2.3 & 99.2 & $2.8(\mathrm{R})$ & APA & 3.8 & 2.6 & normotension \\
\hline 7 & 33 & 7 & moderate & 0 & 2.8 & 82.9 & $2.1(\mathrm{R})$ & APA & 4 & 2.2 & normotension \\
\hline 8 & 31 & 5 & severe & 0 & 2.9 & 34.8 & 0.59 (PRA) & APA & 4.7 & 3.7 & moderate \\
\hline 9 & 28 & 3 & moderate & tiredness & 3.2 & 37.8 & 0.26 (PRA) & APA & 4.5 & 8.6 & normotension \\
\hline 10 & 22 & 3 & severe & 0 & 2.3 & 64.1 & 0.15 (PRA) & APA & 4.1 & 2.5 & normotension \\
\hline 11 & 31 & 7 & moderate & 0 & 2.7 & 73.8 & 0.29 (PRA) & $\mathrm{IHA}$ & & & \\
\hline
\end{tabular}

Table 2 Pregnancy related complications in subjects with primary aldosteronism

\begin{tabular}{|c|c|c|c|c|c|c|c|}
\hline $\begin{array}{c}\text { Patient } \\
\qquad \#\end{array}$ & $\begin{array}{l}\text { Age at gravidity } \\
\text { (years) }\end{array}$ & $\begin{array}{l}\text { Hypertension before } \\
\text { pregnancy (years) }\end{array}$ & Blood pressure during pregnancy & $\begin{array}{c}\text { Gestational week } \\
\text { at delivery }\end{array}$ & Delivery & Complications & $\begin{array}{l}\text { Hypertension } \\
\text { after puerperium }\end{array}$ \\
\hline 1 & 27 & 0 & mild gestational hypertension & 40 & spontaneous & 0 & no \\
\hline 2 & 35 & 0 & mild gestational hypertension & 40 & cesarean section & herpes vaginalis & yes \\
\hline 3 & 28 & 2 & significant BP increase during the $8^{\text {th }}$ month & 36 & cesarean section & discharge of amniotic fluid & yes \\
\hline \multirow[t]{2}{*}{4} & 34 & 1 & mild BP increase & 40 & spontaneous & 0 & yes \\
\hline & 36 & 3 & mild BP increase at the end of gravidity & 41 & spontaneous (induction) & IUGR & yes \\
\hline 5 & 26 & 0 & moderate gestational hypertension & 36 & cesarean section & preeclampsia & yes \\
\hline \multirow[t]{2}{*}{6} & 27 & 0 & mild gestational hypertension & 40 & spontaneous & & yes \\
\hline & 30 & 3 & moderate BP increase & 40 & spontaneous & & yes \\
\hline 7 & 29 & 4 & gradual BP elevation & 27 & cesarean section & preeclampsia & yes \\
\hline 8 & 26 & 3 & moderate BP increase & 39 & cesarean section & preeclampsia & yes \\
\hline 9 & 25 & 0 & BP elevation at the end of gravidity & 39 & cesarean section & preeclampsia & yes \\
\hline 10 & 29 & 5 & BP decompensation during the $2^{\text {nd }}$ trimester & 33 & cesarean section & preeclampsia & yes \\
\hline 11 & 22 & 3 & $\mathrm{BP}$ decompensation during the $2^{\text {nd }}$ trimester & 31 & cesarean section & preeclampsia & yes \\
\hline
\end{tabular}

Apart from hypertension, the most frequent pregnancy-related complication of PA is preeclampsia, sometimes leading to very preterm delivery. The best prevention of these complications is only early diagnosis of PA, in these particular hypertensive cases the awareness of hypokalemia. In some cases, BP elevation during pregnancy was the first presentation of PA. 